\title{
Nuclear Localization Is Not Required for Tip60 Tumor Suppressor Activity in Breast and Lung Cancer Cells
}

\author{
Priyadarshini Ravichandran,, Simon A. Davis,, ${ }^{2, *}$ Himali Vashishtha, ${ }^{3, *}$ \\ Azad L. Gucwa, ${ }^{4}$ and Daniel S. Ginsburg ${ }^{5}$
}

\begin{abstract}
The Tip60 lysine acetyltransferase is a tumor suppressor in most cancers but an oncogene in prostate and gastric cancer. Tip60 is commonly found in the nucleus, where it acetylates proteins involved in transcription, DNA repair, and chromatin; however, it has also been shown to acetylate cytoplasmic targets. In this study, we investigated the relationship between Tip60 localization and breast and lung cancer. In cell fractionation experiments, cancer-derived cell lines showed a shift from nuclear to cytoplasmic endogenous Tip60 compared with cell lines derived from normal cells. With immunofluorescence, we observed four different localization patterns of overexpressed Tip60 and found that cancer cells had increased cytoplasmic localization of Tip60 compared with HEK-293 cells. The addition of a nuclear localization signal (NLS) increased the number of cells containing nuclear Tip60, whereas mutation of a putative endogenous NLS increased the number of cells with cytoplasmic Tip60. Overexpression of Tip60 increased cancer cell line sensitivity to paclitaxel regardless of changes in localization. These results suggest that dysregulation of Tip60 in breast and lung cancer is not limited to reduced expression but may also involve subcellular localization.
\end{abstract}

Keywords: Tip60, localization, tumor suppressor, breast cancer, lung cancer

\section{Introduction}

$\mathbf{T}$ IP60 IS A LYSINE acetyltransferase (KAT) enzyme (Sterner and Berger, 2000) that functions as part of a 1.5 MDa eponymous protein complex (TIP60), containing at least 16 subunits (Doyon et al., 2004). TIP60 is involved in a number of cellular processes including transcription, DNA repair, differentiation, and cell cycle regulation. It serves as a general coactivator at Pol II-transcribed genes (Ravens et al., 2015) and is involved in the regulation of transcription factors including $\mathrm{p} 53$, androgen receptor, and NF- $\mathrm{NB}$ (Tang et al., 2006; Jaganathan et al., 2014). TIP60 is involved in chromatin remodeling and interacts with multiple DNA repair proteins including ATM and DNA-PK (Squatrito et al., 2006). It is recruited to genes involved in stem cell self-renewal and differentiation and represses genes required for differentiation (Fazzio et al., 2008). TIP60 is involved in cell cycle progression, mediating acetylation of p53 to promote apoptosis (Tang et al., 2006). Knockdown of Tip60 disrupts the cell cycle (Zhang et al., 2012), possibly because acetylation of Aurora B promotes accurate chromosome segregation during mitosis (Mo et al., 2016). Tip60 represses telomerase activation by inhibiting the association of the transcriptional regulator Sp1 with the TERT promoter (Rajagopalan et al., 2017).

Consistent with Tip60's role in chromatin alteration, there are two reported nuclear localization signals (NLSs) (Hass and Yankner, 2005) and many studies reporting nuclear localization (McAllister et al., 2002; Dohmesen et al., 2008; Sho et al., 2011; Domingues et al., 2014). Tip60 is recruited to PML bodies by PML3 (Wu et al., 2009), where it interacts with the $\beta$-amyloid precursor protein intracellular domain and FE65, among others (Cao and Südhof, 2001). Phosphorylation of Tip60 Y327 by Abl kinase seems to increase the amount of cytoplasmic Tip60 (a Y327F mutant shows no cytoplasmic localization) (Shin and Kang, 2013). Tip60 in cytoplasmic speckles promotes stabilization of the Nmi protein (Zhang et al., 2007). HeLa cells were found to contain Tip60 primarily in cytoplasmic speckles (Sliva et al., 1999).

Tip60 is associated with multiple diseases, most notably Alzheimer's disease (Johnson et al., 2013) and cancer

\footnotetext{
${ }^{1}$ Feinstein Institute for Medical Research, Manhasset, New York, USA.

${ }^{2}$ New York Structural Biology Center, New York, New York, USA.

${ }^{3}$ VA NY Harbor Health Care System, New York, New York, USA.

${ }^{4}$ Department of Biology, Farmingdale State College, Farmingdale, New York, USA.

${ }^{5}$ Department of Natural Sciences, Immaculata University, Immaculata, Pennsylvania, USA.

*These authors contributed equally to this work.
} 
(Gorrini et al., 2007). In the majority of cancers studied including breast, colorectal, head and neck, skin, and B cell lymphomas, Tip60 functions as a tumor suppressor, consistent with its role in p53 activation. In prostate cancer, Tip60 seems to function as an oncoprotein, promoting resistance to DNA-damaging chemotherapy agents (Coffey et al., 2012) and serving as a coactivator for the androgen

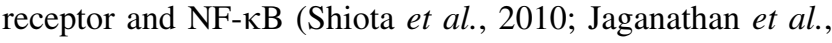
2014).

The connection between Tip60 subcellular localization and its role in cancer is not clear. Nuclear Tip60 staining was reduced in some breast, colon, lung, and stomach cancers, with faint cytoplasmic staining in these cells (Gorrini et al., 2007). Increased nuclear Tip60 correlated with prostate cancer severity (Halkidou et al., 2003). This is consistent with Tip60's role as a tumor suppressor in most cancers but an oncogene in prostate cancer. We were interested in better understanding the role of Tip60 localization in cancer cells.

\section{Materials and Methods}

Tip60 expression plasmid pAW48-FLAG-Tip60-Amp (Peng et al., 2012) was a gift from Dr. Edward Seto (H. Lee Moffitt Cancer Center and Research Institute). NLSs were added to each terminus of Tip60 in pAW48-FLAG-Tip60Amp by amplifying Tip60 from pAW48-FLAG-Tip60-Amp with the primers 5'-GGTACCGAATTCAAGAGACCCGC CGCCACCAAGAAGGCCGGCCAGGCCAAGAAGAAG AAGGCGGAGGTGGTGGAGATAATC-3' and 5'-GGCA AACAACAGATGGCTGG-3' for the N-terminal NLS and 5'-GAACCCACTGCTTACTGGCT-3' and 5'-GATCCTG GTCACCACTTCTTCTTCTTGGCCTGGCCGGCCTTCT TGGTGGCGGCGGGTCTCTTCTTCCCCCTCTTGCTCC AGTC-3' for the C-terminal NLS. PCR products were ligated into pCR2.1-TOPO using the TOPO-TA Cloning Kit (Thermo Fisher, Waltham, MA) according to the manufacturer's instructions to make pTA-FLAG-NLS-Tip60 and pTA-FLAG-Tip60-NLS. Tip60 was removed from pTAFLAG-Tip60-NLS and pTA-FLAG-NLS-Tip60 with EcoRI and $B s t$ EII and cloned into pAW48-FLAG-Tip60-Amp from which Tip60 had been removed to make pAW48-FLAGNLS-Tip60 and pAW48-FLAG-Tip60-NLS.

pAW48-FLAG-Tip60-mutNLS was generated by sitedirected mutagenesis of pAW48-FLAG-Tip60-Amp with a QuikChange II XL Site-Directed Mutagenesis Kit (Agilent Technologies, Santa Clara, CA) according to the manufacturer's instructions using primers 5'-GGCAGCCCAGCC AGGACAGCAGCAAAACTCGAATTGTTTGGGCAC-3' and 5'-GTGCCCAAACAATTCGAGTTTTGCTGCTGTCC TGGCTGGGCTGCC-3'. All plasmids were confirmed by restriction analysis and sequencing.

Cells used in this study (A549, H358, NL20, BT474, SKBR-3, MDA-MB-231, MCF10A, SW1990, PL45, and HEK293) were cultured in Dulbecco's modified Eagle's medium, with $1 \%$ L-glutamine, $1 \%$ penicillin-streptomycin, and $10 \%$ fetal bovine serum (Thermo Fisher) in a humidified incubator with $5 \% \mathrm{CO}_{2}$ at $37^{\circ} \mathrm{C}$. Transfections were performed with Xfect Transfection Reagent (Takara Bio USA, Inc., Mountain View, CA) or TransIT-X2 (Mirus Bio LLC, Madison, WI) according to the manufacturer's protocol. Cell fractionation was conducted with the NE-PER Nuclear and Cytoplasmic Extraction Reagents Kit (Thermo Fisher) according to the manufacturer's instructions. IRB approval was obtained prior to beginning these studies. For wholecell extracts, cells were washed three times in phosphatebuffered saline (150 mM NaCl, $20 \mathrm{mM}$ phosphate buffer, $\mathrm{pH}$ 7.4) and then lysed in lysis buffer $(50 \mathrm{mM}$ Tris, $\mathrm{pH} 8.0$, $150 \mathrm{mM} \mathrm{NaCl}, 1 \%$ Triton X-100) with a protease inhibitor cocktail and phosphatase inhibitors $(10 \mathrm{mM} \mathrm{NaF}, 10 \mathrm{mM}$ $\mathrm{Na}_{3} \mathrm{VO}_{4}$ ) (Thermo Fisher). Cells were lysed on ice for $20 \mathrm{~min}$, scraped, and spun down at $4^{\circ} \mathrm{C}$ for $15 \mathrm{~min}$. Protein concentration was measured by Bio-Rad Protein Assay (Bio-Rad, Hercules, CA) and cell viability with WST-1 reagent (Roche, Indianapolis, IN) according to the manufacturers' directions.

Immunofluorescence (IF) was performed as previously described (Gucwa and Brown, 2014) and mounted with Vectashield mounting media containing 4',6-diamidino-2phenylindole (Vector Laboratories, Burlingame, CA). Images were acquired with a $100 \times$ oil immersion objective using a Zeiss Axiovert $40 \mathrm{CFL}$ inverted three-channel fluorescence microscope and processed using Zeiss software (Zen, blue edition).

Antibodies used for Western blotting or IF include rabbit polyclonal anti-KAT5 (ab23886; Abcam), rabbit polyclonal anti-histone $\mathrm{H} 3$ (ab1791; Abcam), mouse ANTI-FLAG ${ }^{\circledR}$ M2 antibody (F3165; Millipore Sigma), rabbit anti-GAPDH (G9545; Millipore Sigma), Alexa Fluor (AF)-488, 594conjugated goat anti-mouse, and goat anti-rabbit IgG antibodies (Thermo Fisher).

\section{Results}

Tip60 is considered a tumor suppressor in most cancers. We hypothesized that Tip60 dysfunction in cancer is a combination of reduced expression and activity and/or mislocalization. To test this, we examined Tip60 localization in breast and lung cancer cell lines and whether altering Tip60 localization would affect proliferation.

\section{Cancer cells have more cytoplasmic Tip60 than noncancer cells}

To better understand the role of Tip60 mislocalization in cancer, we performed cell fractionation and analyzed endogenous Tip60 levels in nuclear and cytoplasmic extracts of lung and breast cancer (A549, H358, BT474, SK-BR-3, and MDA-MB-231) and noncancer (NL20 and MCF10A) cell lines. The extracts were subjected to Western blotting with antibodies against Tip60 and loading controls (histone H3 or GAPDH) (Fig. 1A, C). No cancer cells had more nuclear Tip60 than noncancer cells, whereas noncancer cells had significantly more nuclear Tip60 than at least one of the corresponding cancer cell lines (Fig. 1B).

We observed more cytoplasmic Tip60 in cancer cells than in noncancer-derived cells (Fig. 1D). All the cancer cell lines, except SK-BR-3, had significantly more cytoplasmic Tip60 compared with the corresponding noncancer cells. The ratios of nuclear to cytoplasmic Tip60 were significantly higher ( $\geq 2$-fold) in noncancer cells than all the corresponding cancer cell lines (Fig. 1E). A similar pattern of Tip60 localization was seen comparing pancreatic cancer cell lines SW1990 and PL45 with embryonic kidney HEK293 cells and white blood cells (Supplementary Fig. S1). 


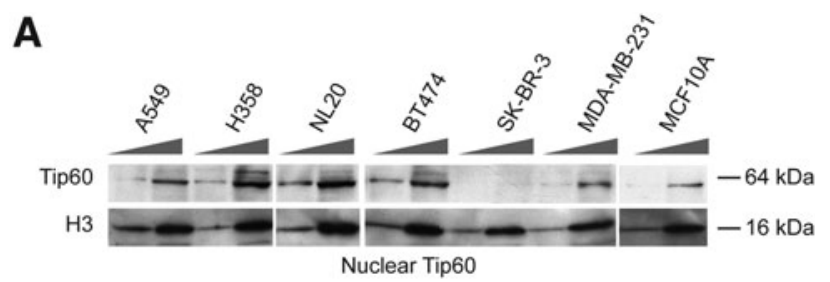

B
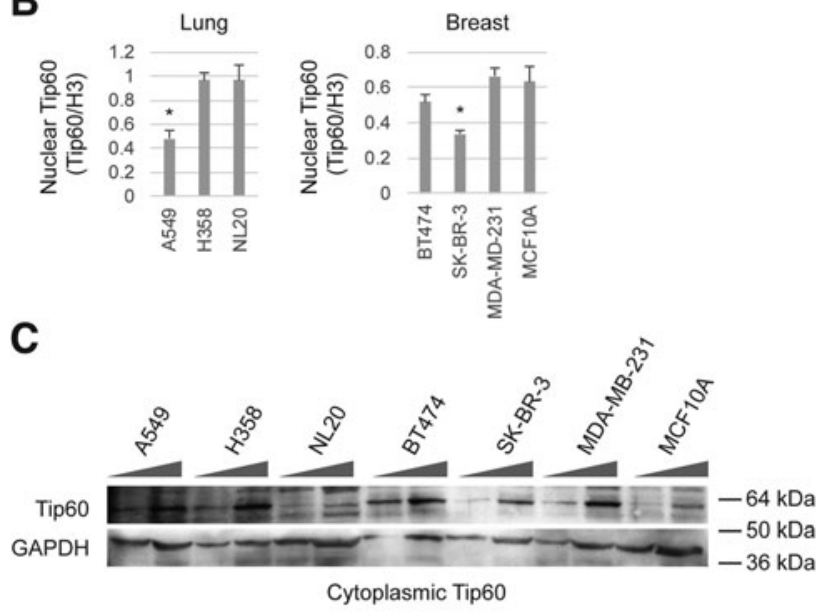

D
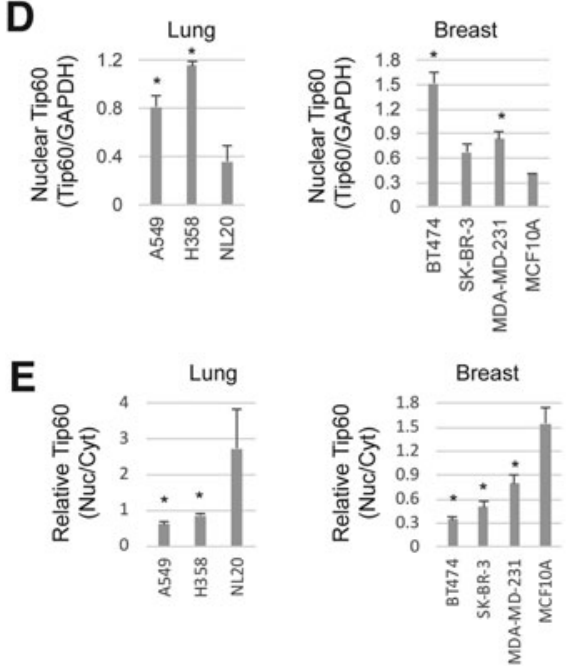

FIG. 1. Cancer cells have more cytoplasmic Tip60 than noncancer-derived cells. Nuclear (A) and cytoplasmic (C) extracts were made from the indicated cells and subjected to Western blotting analysis with anti-Tip60 and anti-H3 or antiGAPDH antibodies. (B, D) Films were scanned and band intensity was quantitated using ImageJ or ImageQuant TL. Relative Tip60 levels were calculated as the ratio of the signal of Tip60 to that of the loading control (H3 or GAPDH). Columns represent an average of at least three independent cultures. Error bars show the standard deviation. A549 and SK-BR-3 cells had significantly less nuclear Tip60 than NL20 and MCF10A cells, respectively, as determined by ANOVA $\left({ }^{*} p<0.01\right)$. A549, H358, BT474, and MDA-MB-231 cells had significantly more cytoplasmic Tip60 than NL20 and MCF10A cells as determined by ANOVA $(* p<0.01)$. (E) The ratio of nuclear to cytoplasmic Tip60 was calculated by dividing the relative nuclear Tip60 level in (B) by the relative cytoplasmic Tip60 level in (D). All the cancer-derived cell lines had a significantly higher ratio of nuclear:cytoplasmic Tip60 than the noncancer-derived controls as determined by ANOVA $(* p<0.01)$. ANOVA, analysis of variance.
This difference suggests that there is a change in Tip60 localization in the cancer cell lines we studied.

\section{Tip60 exhibits different localization patterns}

We examined Tip60 localization by IF in A549, BT474, MDA-MB-231, and HEK-293 cells. We detected overexpressed FLAG-Tip60 with anti-FLAG antibodies because we were unable to detect the low levels of endogenous Tip60 by IF. While most previous studies have reported finding Tip60 in nuclear speckles, we found four major Tip60 localization patterns: diffuse nuclear (homogeneous Tip60 throughout the nucleus), nuclear speckled (staining in nuclear puncta), cytoplasmic (staining throughout the cell excluding the nucleus), and both (staining through the nucleus and cytoplasm) (Fig. 2A).

There were dramatic differences in the distribution of Tip60 localization between HEK-293 cells and the cancerderived cells (Fig. 2B). Approximately 75\% of HEK-293 cells contained Tip60 in nuclear speckles. However, only $\sim 30 \%$ of cancer cells had Tip60 in nuclear speckles. Only $\sim 20 \%$ of HEK-293 cells had Tip60 in the cytoplasm, whereas $30-50 \%$ of the cancer cells had cytoplasmic Tip60. These results are consistent with the cell fractionation experiments, suggesting that cytoplasmic Tip60 increases in the cancer cells we studied.

\section{Tip60 localization can be altered by adding or removing NLSs}

We attempted to increase nuclear localization with the addition of the nucleoplasmin bipartite NLS to both termini of the protein. Constructs containing CMV promoter-driven FLAG-NLS-Tip60 or FLAG-Tip60-NLS were transfected into HEK-293, A549, MDA-MB-231, and BT474 cells. Neither NLS addition reduced Tip60 expression in HEK293 cells (Fig. 3A). Tip60 localization was determined by IF with anti-FLAG antibodies.

The addition of an NLS significantly increased nuclear localization of Tip60 in all the cell lines (Fig. 3B). Cancer cells showed a $20-40 \%$ increase in the number of cells without cytoplasmic Tip60, with the greatest effect observed in MDA-MB-231 cells. The smallest effect was seen in HEK-293 cells, consistent with the finding that these cells had the least cytoplasmic Tip60. Interestingly, the N-terminal and C-terminal NLSs had cell line-specific effects. In HEK293 cells, the C-terminal NLS had a larger effect than the N-terminal NLS, whereas we observed the opposite phenomenon in MDA-MB-231 cells. Localization was unaffected by NLS location in BT474 and A549 cells.

We also attempted to increase cytoplasmic Tip60 by mutating one of the putative NLSs (changing position 217 from PGRKRKS to PGQQQNS). The mutation did not affect Tip60 expression (Fig. 4A). Overexpression of the mutant protein led to significantly fewer cells with Tip60 only in the nucleus and more cells with Tip60 in the cytoplasm in all four cell lines (Fig. 4B). Cytoplasmic Tip60 localization increased from $\sim 50 \%$ to $100 \%$, depending on the cell line, suggesting that this sequence is an NLS. Nuclear Tip60 was not eliminated completely, consistent with the second putative bipartite NLS reported at position 328 (Hass and Yankner, 2005). 


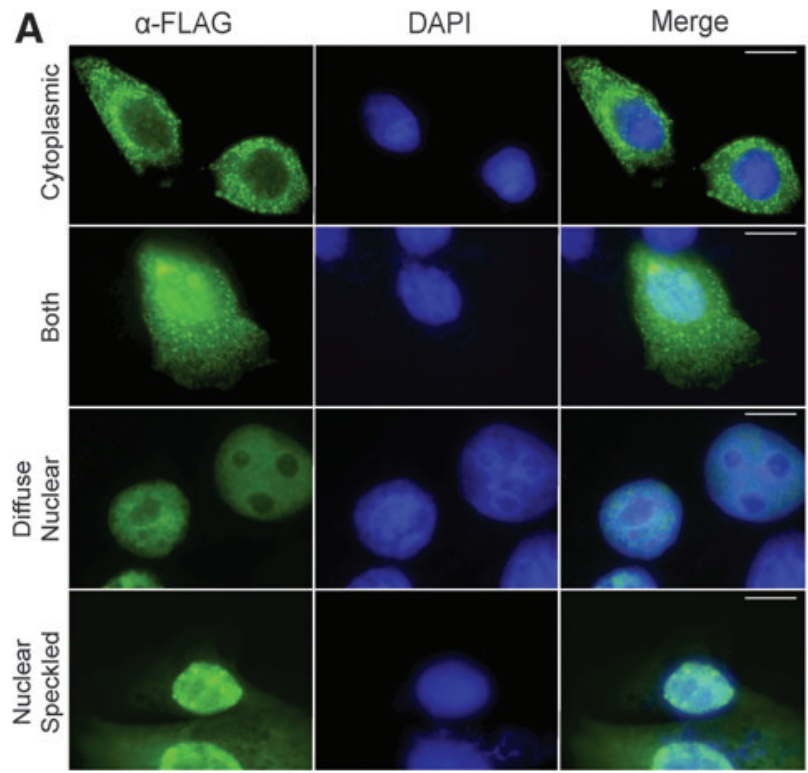

B

FIG. 2. Tip60 displays four different localization patterns. (A) Cells were transfected with pAW48-FLAG-Tip60-Amp and processed for immunofluorescence $48 \mathrm{~h}$ later using anti-FLAG antibodies. DAPI (blue) was used to stain nuclei. (B) The indicated cell lines were treated as in (A), and then the number of cells with each type of Tip60 localization was counted ( 600 cells total for each cell line). A549, BT474, and MDA-MB-231 cancer cells have significantly different distributions of Tip60 localization compared with embryonic HEK-293 cells as determined by $\chi^{2}$ test $(* p<0.001)$.

Overexpression of Tip60 can reduce proliferation of breast and lung cancer cells independent of localization

Because Tip60 is considered a tumor suppressor in breast (Bassi et al., 2016) and lung (Eymin et al., 2006) cancer, we hypothesized that overexpression of the KAT could lead to reduced proliferation in cancer cell lines in combination with chemotherapy. Overexpression of Tip60 alone did not affect the growth of lung or breast cancer cell lines (Supplementary Fig. S2). To test the effect of Tip60 in combination with chemotherapy, we transfected A549,

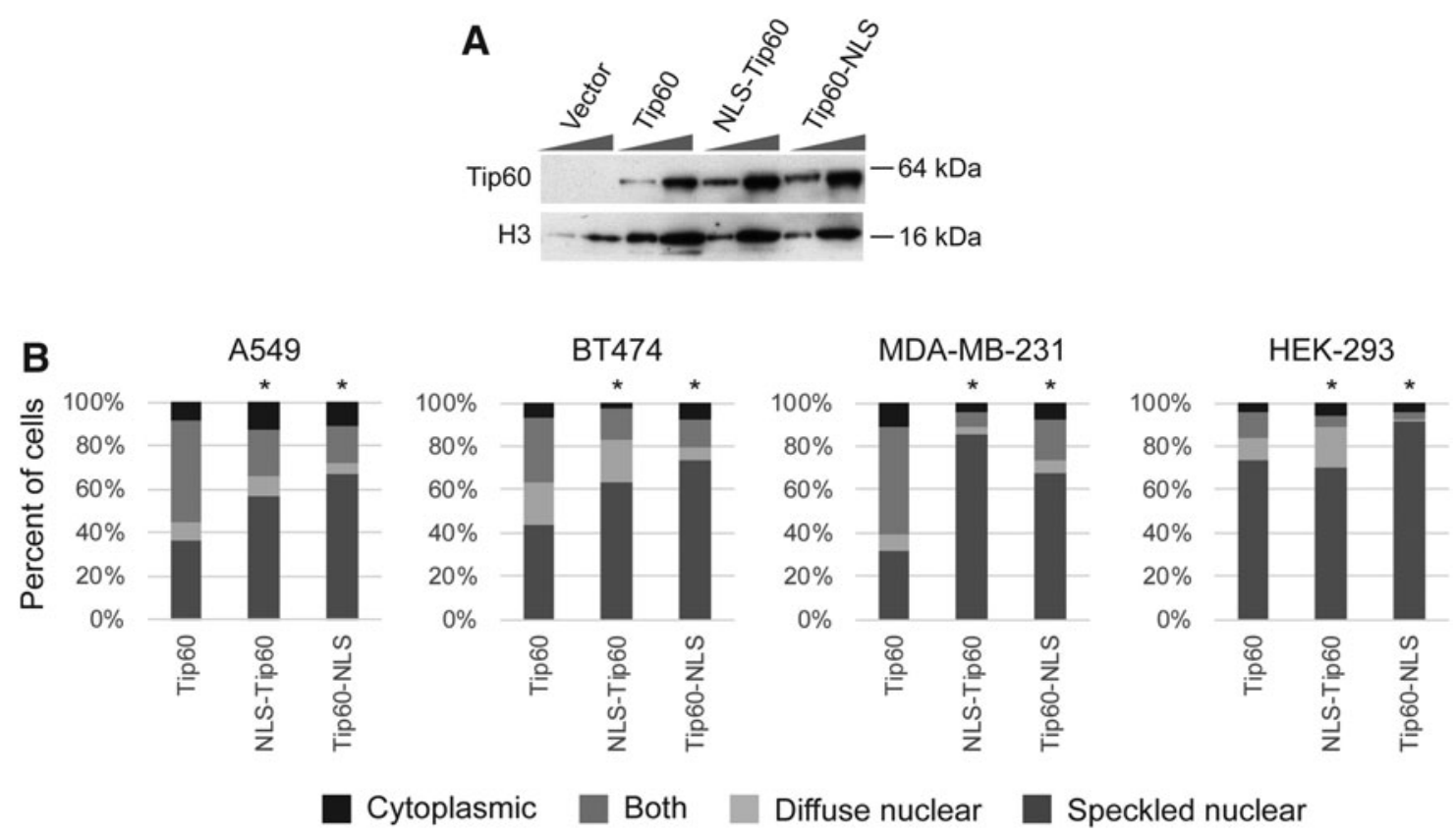

FIG. 3. Adding NLSs to Tip60 increases nuclear localization. (A) Tip60 expression in HEK-293 cells. HEK-293 cells were transfected with pcDNA3.1, pAW48-FLAG-Tip60-Amp, pAW48-FLAG-NLS-Tip60, and pAW48-FLAG-Tip60-NLS, and whole-cell extracts were subjected to Western blotting analysis with anti-Tip60 and anti-histone H3 antibodies. (B) Tip60 localization patterns. The indicated cell lines were transfected with pAW48-FLAG-Tip60-Amp, pAW48-FLAG-NLS-Tip60, and pAW48-FLAG-Tip60-NLS and treated as in Figure 2B ( 300 cells counted per plasmid). The addition of an NLS significantly increased nuclear Tip60 localization as determined by $\chi^{2}$ test $\left({ }^{*} p<0.001\right)$. NLS, nuclear localization signal. 


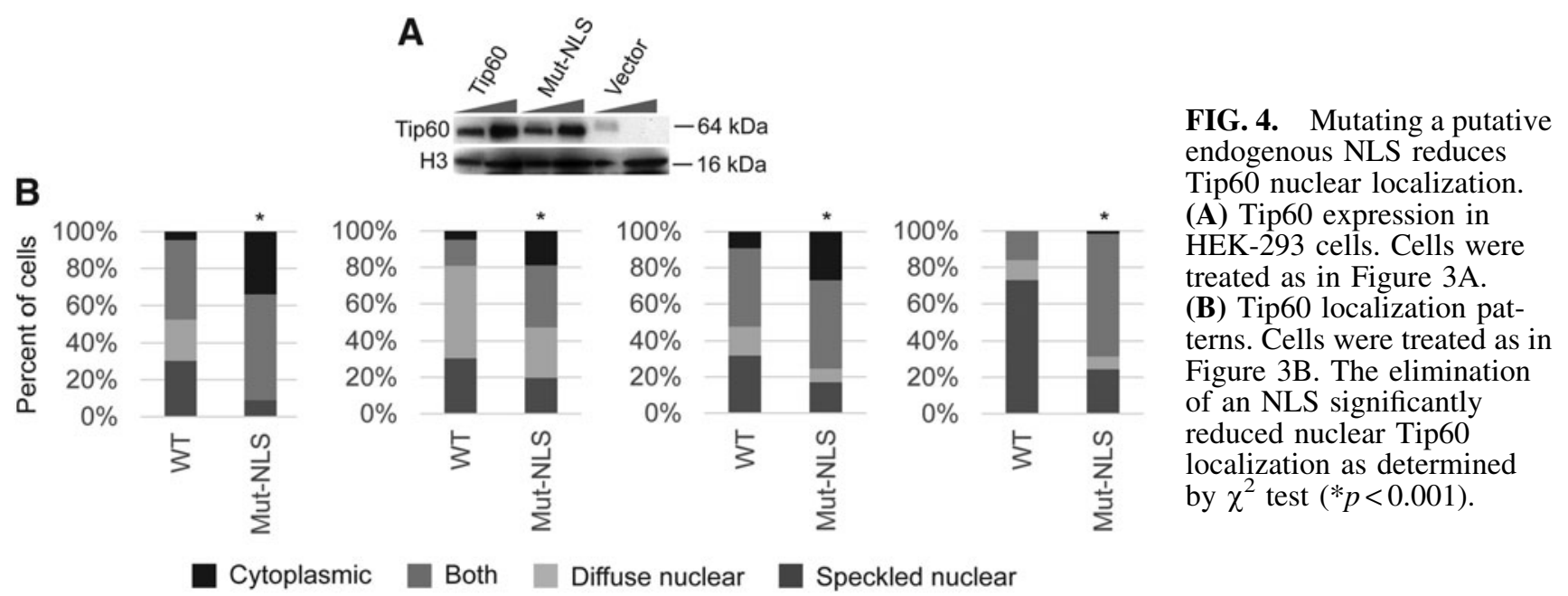

BT474, MDA-MB-231, and HEK-293 cells with plasmids expressing WT Tip60, Tip60-NLS, or Tip60-Mut-NLS, treated the cells with paclitaxel, and measured cellular proliferation over $48 \mathrm{~h}$.

Overexpression of Tip60 significantly reduced proliferation of cancer cells in the presence of paclitaxel (Fig. 5A-C).
In A549 cells, there were $\sim 25 \%$ fewer Tip60-transfected cells than cells transfected with an empty vector after $48 \mathrm{~h}$ (Fig. 5A). In BT474 cells, overexpression of Tip60 significantly reduced the number of viable cells by $\sim 65 \%$ after $48 \mathrm{~h}$ compared with cells without Tip60 overexpression (Fig. 5B). Tip60 reduced proliferation of MDA-MB-231 cells
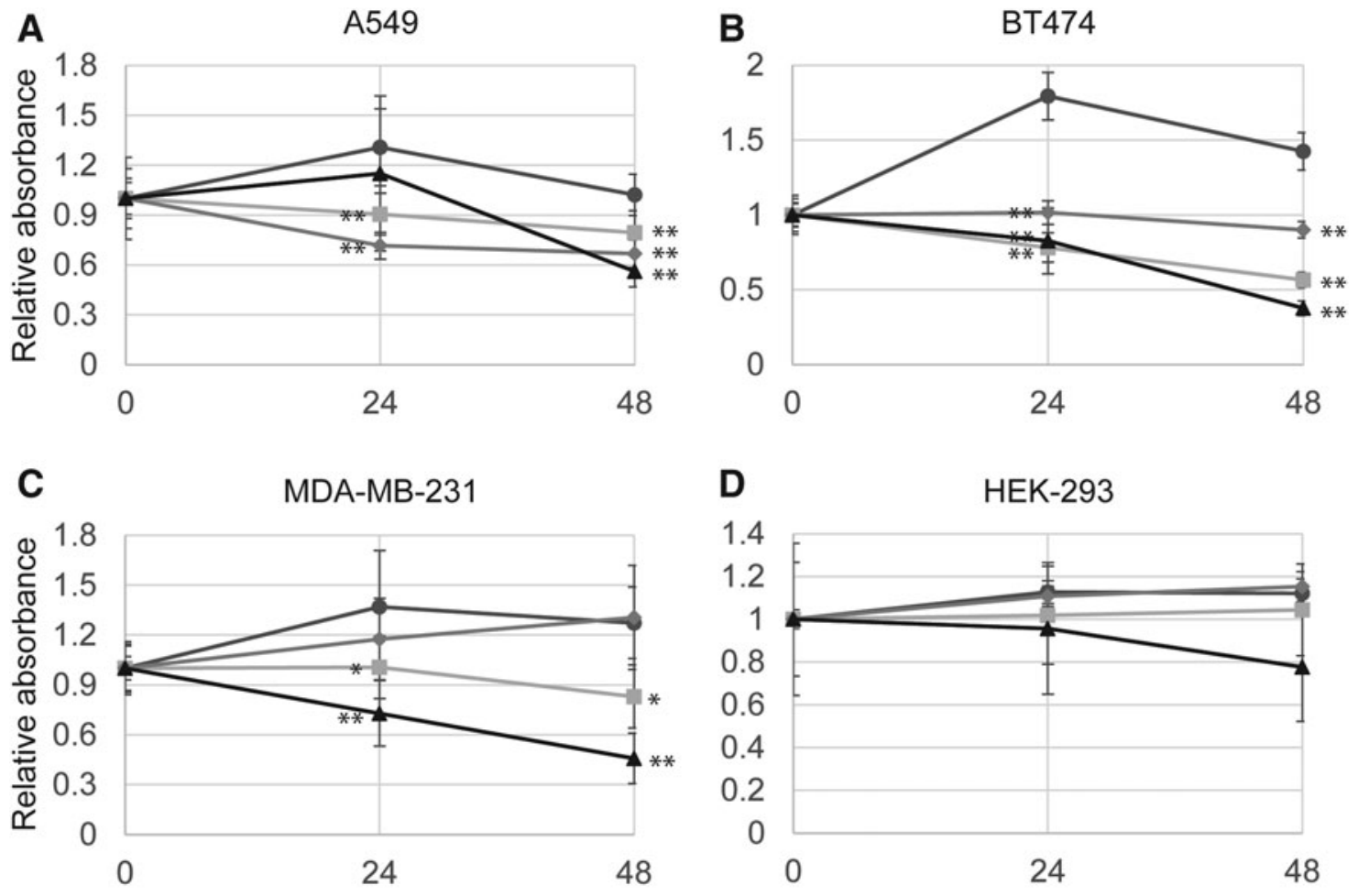

Hours in paclitaxel

D

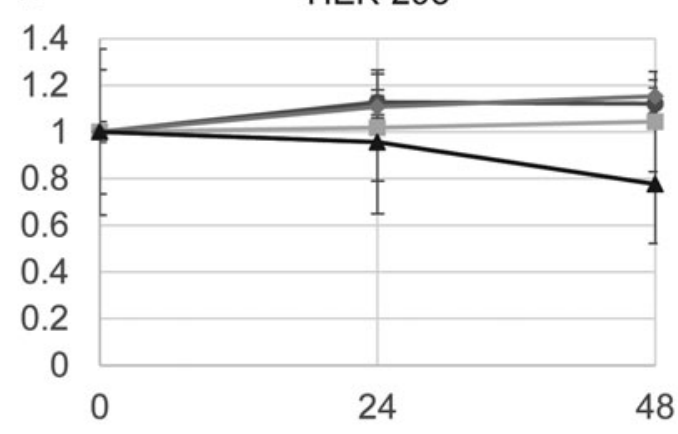

Hours in paclitaxel

\section{Tip60-NLS $\Delta$ Tip60-Mut-NLS}

FIG. 5. Tip60 overexpression reduces proliferation of cancer-derived cells. A549 (A), BT474 (B), MDA-MB-231 (C), and HEK-293 (D) cells were transfected with pcDNA3.1, pAW48-FLAG-Tip60-Amp, pAW48-FLAG-Tip60-NLS, and pAW48-FLAG-Tip60-Mut-NLS, treated with paclitaxel after $48 \mathrm{~h}$, and cell viability was determined by WST-1 assay at the indicated time points. For each plasmid, the absorbance at 24 and $48 \mathrm{~h}$ was normalized to the absorbance at $0 \mathrm{~h}$ after subtracting out the background. Tip60 overexpression significantly reduced proliferation of A549, BT474, and MDA-MB231 cells as determined by ANOVA $(* p<0.05, * * p<0.01)$. 
by $\sim 35 \%$ after $48 \mathrm{~h}$ (Fig. 5C). Tip60 overexpression did not significantly affect proliferation of HEK-293 cells (Fig. 5D).

The effects of Tip60 on cellular proliferation appeared to be independent of localization. We expected increased nuclear Tip60 (Tip60-NLS) to have a greater antiproliferative effect and increased cytoplasmic Tip60 (Tip60-Mut-NLS) to have a reduced antiproliferative effect compared with WT. Surprisingly, all three Tip60 constructs resulted in similar reductions in proliferation in A549 and BT474 cells (Fig. 5A, B), even though the addition or removal of an NLS significantly altered Tip60's localization (Fig. 3). In MDAMB-231 cells, Tip60-NLS had no effect on proliferation, whereas Tip60-Mut-NLS had the greatest effect on proliferation (Fig. 5C). These results suggest that Tip60 antiproliferative functions take place in both the cytoplasm and the nucleus and that Tip60 may have some cell type-specific roles or that there are other players involved.

\section{Discussion}

Tip60 expression is inversely correlated with increased tumor size and progression and decreased patient survival rates (Sakuraba et al., 2009, 2011; Chen et al., 2012). Consistent with its roles in transcription and DNA repair, Tip60 is most often found in the nucleus but has also been found in the cytoplasm (Shin and Kang, 2013). The focus of this study was to understand the relationship between Tip60 localization and cancer. We have provided multiple lines of evidence that Tip60 localization is altered in the breast and lung cancer cells we studied.

First, we showed that breast, lung, and pancreatic cancer cell lines have a significantly different Tip60 subcellular localization distribution compared with noncancer-derived cell lines (Figs. 1E, S1). The cancer cell lines had less nuclear Tip60 (Fig. 1A, B) than the corresponding normal cell lines. The differences were more dramatic for cytoplasmic Tip60, with all cancer cell lines except SK-BR-3 having significantly more cytoplasmic Tip60 than the corresponding normal cell lines (Fig. 1C, D). This suggests that a mislocalization of Tip60 occurred in these cancer cells, although the mechanism is not clear. The altered localization may be a contributing factor in tumorigenesis in combination with reduced Tip60 expression.

We also observed that overexpressed Tip60 localized differently in cancer-derived cell lines compared with HEK293 cells (Fig. 2). When we overexpressed Tip60, we observed four different localization patterns. The nuclear speckles are likely to be PML bodies as has been previously described (Wu et al., 2009). The other localization patterns may be cell cycle dependent or due to stochastic changes in Tip60 expression. Because PML bodies are thought to be involved in transcription, genome organization, and DNA repair (Dundr, 2012), it is possible that diffuse nuclear Tip60 staining is found in senescent cells with limited transcriptional activity. In all the cell types we looked at the majority of cells (70-90\%) contained nuclear Tip60. Although a majority of the cancer-derived cells still contained Tip60 in the nucleus, the number of cells with Tip60 in the cytoplasm was significantly greater than in HEK-293 cells. This is consistent with our observations of endogenous Tip60 that cancer-derived cells have increased cytoplasmic Tip60.
Since Tip60 is a tumor suppressor in breast and lung cancer (Eymin et al., 2006; Bassi et al., 2016), we hypothesized that overexpression of Tip60 in these cancer cells would decrease their proliferation. After we observed that cancer-derived cells have increased cytoplasmic Tip60, we also tested whether changing Tip60 localization would affect cancer cell growth. We first found that Tip60 localization could be altered. The addition of an NLS significantly increased the number of cells with nuclear Tip60 in all cell lines tested (Fig. 3). Conversely, mutation of a putative endogenous NLS significantly increased the number of cells with cytoplasmic Tip60 (Fig. 4).

While the addition of an NLS to either the amino or carboxyl terminus of Tip60 increased the number of cells with nuclear Tip60, the position of the NLS did seem to matter in a cell line-specific manner. In A549 and HEK-293 cells, the C-terminal NLS had the greatest effect, whereas in the other two cell lines, the N-terminal NLS resulted in the most cells with nuclear Tip60. We propose that the most effective NLS is on the terminus that is more exposed to the nuclear import machinery. It is possible that Tip60 is interacting with different proteins in the different cell lines, partially masking one terminus or the other.

The finding that positive amino acids in the putative NLS starting at position 217 significantly reduced the number of cells with nuclear Tip60 strongly suggests that this sequence functions as an NLS. The Tip60 remaining in the nucleus is likely due to the other proposed bipartite NLS. The presence of two NLSs is consistent with the fact that the majority of cells overexpressing WT Tip60 contain nuclear Tip60.

We next asked whether overexpression of Tip60 would slow the growth of cancer cells. Overexpression of Tip60 on its own had no effect on the growth of A549, BT474, MDAMB-231, and HEK-293 cells (Supplementary Fig. S2), suggesting that while loss of Tip60 may contribute to cancer, it is insufficient for transforming cells. This is consistent with the fact that loss of Tip60 expression is often found in conjunction with p53 mutations (Gorrini et al., 2007).

Because Tip60 overexpression had no effect on its own, we analyzed whether Tip60 overexpression could sensitize cells to paclitaxel. Tip60 overexpression in combination with paclitaxel significantly reduced the growth of the cancer cell lines, but not HEK-293 cells (Fig. 5). Because cancer-derived cells had increased cytoplasmic Tip60, we expected that increased nuclear Tip60 would further reduce cancer cell growth and increased cytoplasmic Tip60 would affect growth less than WT Tip60. Surprisingly, we found that all three constructs behaved similarly in A549 and BT474 cells. In MDA-MB-231 cells, Tip60-NLS had no effect on growth.

Tip60 acetylates p53 and serves as a coactivator at p53 target genes in the nucleus (Legube et al., 2004). Tip60 has also been shown to acetylate cortactin in the cytoplasm to inhibit breast cancer cell migration and invasion (Sun et al., 2015). Thus, Tip60 has tumor suppressor functions in both the nucleus and the cytoplasm, suggesting that localization did not affect cell growth because Tip60 can function as a tumor suppressor in both cellular compartments. While we significantly altered the distribution of Tip60 localization, we did not eliminate either nuclear or cytoplasmic Tip60 and perhaps did not have enough overexpression to affect cellular proliferation. 


\section{Conclusions}

Tip60 has been viewed as a therapeutic target in prostate and gastric cancer because it is overexpressed in those cancers and contributes to resistance to DNA-damaging chemotherapeutic drugs (Coffey et al., 2012; Shi et al., 2016). In most other cancers, including the breast and lung cancers studied here, Tip60 is thought to be a tumor suppressor. We have demonstrated that in addition to lower expression, Tip60 localization is also changed in cancer cells. We have confirmed one of the two putative NLSs in Tip60 and shown that Tip60 overexpression can reduce proliferation of breast and lung cancer cells. Understanding the relationship between Tip60 localization and cancer may allow for new therapies that target not only KAT activity but also Tip60 expression and localization.

\section{Acknowledgment}

We thank Dr. Edward Seto (H. Lee Moffitt Cancer Center and Research Institute) for pAW48-FLAG-Tip60-Amp.

\section{Authors' Contributions}

P.R. conceived the project along with D.S.G., performed experiments and analyzed data with WT Tip60 and NLSTip60, and wrote the article with D.S.G and A.L.G. S.A.D. performed experiments and analyzed data with Tip60-NLS. H.V. performed experiments and analyzed data with Tip60Mut-NLS. A.L.G. helped perform and analyze data from immunofluorescence experiments. D.S.G. performed some Western blotting analyses and supervised the work of P.R., S.A.D., and H.V.

\section{Disclosure Statement}

No competing financial interests exist.

\section{Funding Information}

This work was funded through intramural funds from the corresponding authors' institutions.

\section{Supplementary Material}

Supplementary Figure S1

Supplementary Figure S2

\section{References}

Bassi, C., Li, Y.T., Khu, K., Mateo, F., Baniasadi, P.S., Elia, A., et al. (2016). The acetyltransferase Tip60 contributes to mammary tumorigenesis by modulating DNA repair. Cell Death Differ 23, 1198-1208.

Cao, X., and Südhof, T.C. (2001). A transcriptionally [correction of transcriptively] active complex of APP with Fe65 and histone acetyltransferase Tip60. Science 293, 115-120.

Chen, G., Cheng, Y., Tang, Y., Martinka, M., and Li, G. (2012). Role of Tip60 in human melanoma cell migration, metastasis, and patient survival. J Invest Dermatol 132, 2632-2641.

Coffey, K., Blackburn, T.J., Cook, S., Golding, B.T., Griffin, R.J., Hardcastle, I.R., et al. (2012). Characterisation of a Tip60 specific inhibitor, NU9056, in prostate cancer. PLoS One 7, e45539.
Dohmesen, C., Koeppel, M., and Dobbelstein, M. (2008). Specific inhibition of Mdm2-mediated neddylation by Tip60. Cell Cycle 7, 222-231.

Domingues, S.C., Konietzko, U., Henriques, A.G., Rebelo, S., Fardilha, M., Nishitani, H., et al. (2014). RanBP9 modulates AICD localization and transcriptional activity via direct interaction with Tip60. J Alzheimers Dis 42, 1415-1433.

Doyon, Y., Selleck, W., Lane, W.S., Tan, S., and Côté J. (2004). Structural and functional conservation of the NuA4 histone acetyltransferase complex from yeast to humans. Mol Cell Biol 24, 1884-1896.

Dundr, M. (2012). Nuclear bodies: multifunctional companions of the genome. Curr Opin Cell Biol 24, 415-422.

Eymin, B., Claverie, P., Salon, C., Leduc, C., Col, E., Brambilla, E., et al. (2006). p14ARF activates a Tip60-dependent and $\mathrm{p} 53$-independent ATM/ATR/CHK pathway in response to genotoxic stress. Mol Cell Biol 26, 4339-4350.

Fazzio, T.G., Huff, J.T., and Panning, B. (2008). An RNAi screen of chromatin proteins identifies Tip60-p400 as a regulator of embryonic stem cell identity. Cell 134, 162-174.

Gorrini, C., Squatrito, M., Luise, C., Syed, N., Perna, D., Wark, L., et al. (2007). Tip60 is a haplo-insufficient tumour suppressor required for an oncogene-induced DNA damage response. Nature 448, 1063-1067.

Gucwa, A.L., and Brown, D.A. (2014). UIM domain-dependent recruitment of the endocytic adaptor protein Eps15 to ubiquitin-enriched endosomes. BMC Cell Biol 15, 34.

Halkidou, K., Gnanapragasam, V.J., Mehta, P.B., Logan, I.R., Brady, M.E., Cook, S., et al. (2003). Expression of Tip60, an androgen receptor coactivator, and its role in prostate cancer development. Oncogene 22, 2466-2477.

Hass, M.R., and Yankner, B.A. (2005). A \{gamma\}-secretaseindependent mechanism of signal transduction by the amyloid precursor protein. J Biol Chem 280, 36895-36904.

Jaganathan, A., Chaurasia, P., Xiao, G.Q., Philizaire, M., Lv, X., Yao, S., et al. (2014). Coactivator MYST1 regulates nuclear factor- $\kappa \mathrm{B}$ and androgen receptor functions during proliferation of prostate cancer cells. Mol Endocrinol 28, 872-885.

Johnson, A.A., Sarthi, J., Pirooznia, S.K., Reube, W., and Elefant, F. (2013). Increasing Tip60 HAT levels rescues axonal transport defects and associated behavioral phenotypes in a Drosophila Alzheimer's disease model. J Neurosci 33, 7535-7547.

Legube, G., Linares, L.K., Tyteca, S., Caron, C., Scheffner, M., Chevillard-Briet, M., et al. (2004). Role of the histone acetyl transferase Tip60 in the p53 pathway. J Biol Chem 279, 44825-44833.

McAllister, D., Merlo, X., and Lough, J. (2002). Characterization and expression of the mouse tat interactive protein $60 \mathrm{kD}$ (TIP60) gene. Gene 289, 169-176.

Mo, F., Zhuang, X., Liu, X., Yao, P.Y., Qin, B., Su, Z., et al. (2016). Acetylation of Aurora B by TIP60 ensures accurate chromosomal segregation. Nat Chem Biol 12, 226-232.

Peng, L., Ling, H., Yuan, Z., Fang, B., Bloom, G., Fukasawa, K., et al. (2012). SIRT1 negatively regulates the activities, functions, and protein levels of hMOF and TIP60. Mol Cell Biol 32, 2823-2836.

Rajagopalan, D., Pandey, A.K., Xiuzhen, M.C., Lee, K.K., Hora, S., Zhang, Y., et al. (2017). TIP60 represses telomerase expression by inhibiting Sp1 binding to the TERT promoter. PLoS Pathog 13, e1006681.

Ravens, S., Yu, C., Ye, T., Stierle, M., and Tora, L. (2015). Tip60 complex binds to active Pol II promoters and a subset 
of enhancers and co-regulates the c-Myc network in mouse embryonic stem cells. Epigenetics Chromatin 8, 45.

Sakuraba, K., Yasuda, T., Sakata, M., Kitamura, Y.H., Shirahata, A., Goto, T., et al. (2009). Down-regulation of Tip60 gene as a potential marker for the malignancy of colorectal cancer. Anticancer Res 29, 3953-3955.

Sakuraba, K., Yokomizo, K., Shirahata, A., Goto, T., Saito, M., Ishibashi, K., et al. (2011). TIP60 as a potential marker for the malignancy of gastric cancer. Anticancer Res 31, 77-79.

Shi, M., Lu, X.J., Zhang, J., Diao, H., Li, G., Xu, L., et al. (2016). Oridonin, a novel lysine acetyltransferases inhibitor, inhibits proliferation and induces apoptosis in gastric cancer cells through p53- and caspase-3-mediated mechanisms. Oncotarget 7, 22623-22631.

Shin, S.H., and Kang, S.S. (2013). Phosphorylation of Tip60 Tyrosine 327 by Abl Kinase Inhibits HAT Activity through Association with FE65. Open Biochem J 7, 66-72.

Shiota, M., Yokomizo, A., Masubuchi, D., Tada, Y., Inokuchi, J., Eto, M., et al. (2010). Tip60 promotes prostate cancer cell proliferation by translocation of androgen receptor into the nucleus. Prostate 70, 540-554.

Sho, T., Tsukiyama, T., Sato, T., Kondo, T., Cheng, J., Saku, T., et al. (2011). TRIM29 negatively regulates p53 via inhibition of Tip60. Biochim Biophys Acta 1813, 1245-1253.

Sliva, D., Zhu, Y.X., Tsai, S., Kamine, J., and Yang, Y.C. (1999). Tip60 interacts with human interleukin-9 receptor alpha-chain. Biochem Biophys Res Commun 263, 149-155.

Squatrito, M., Gorrini, C., and Amati, B. (2006). Tip60 in DNA damage response and growth control: many tricks in one HAT. Trends Cell Biol 16, 433-442.

Sterner, D.E., and Berger, S.L. (2000). Acetylation of histones and transcription-related factors. Microbiol Mol Biol Rev 64, 435-459.

Sun, Y., Sun, J., Lungchukiet, P., Quarni, W., Yang, S., Zhang, $\mathrm{X}$., et al. (2015). Fe65 suppresses breast cancer cell migration and invasion through Tip60 mediated cortactin acetylation. Sci Rep 5, 11529.
Tang, Y., Luo, J., Zhang, W., and Gu, W. (2006). Tip60dependent acetylation of p53 modulates the decision between cell-cycle arrest and apoptosis. Mol Cell 24, 827-839.

Wu, Q., Hu, H., Lan, J., Emenari, C., Wang, Z., Chang, K.S., et al. (2009). PML3 orchestrates the nuclear dynamics and function of TIP60. J Biol Chem 284, 8747-8759.

Zhang, K., Zheng, G., and Yang, Y.C. (2007). Stability of Nmi protein is controlled by its association with Tip60. Mol Cell Biochem 303, 1-8.

Zhang, S.M., Song, M., Yang, T.Y., Fan, R., Liu, X.D., and Zhou, P.K. (2012). HIV-1 Tat impairs cell cycle control by targeting the Tip60, Plk1 and cyclin B1 ternary complex. Cell Cycle 11, 1217-1234.

Address correspondence to:

Daniel S. Ginsburg, PhD

Department of Natural Sciences Immaculata University 1145 King Road Immaculata, PA 19345

USA

E-mail: dginsburg@immaculata.edu

Azad L. Gucwa, PhD Department of Biology Farmingdale State College 2350 Broadhollow Road Farmingdale, NY 11735

USA

E-mail: azad.gucwa@farmingdale.edu

Received for publication July 28, 2020; accepted August 24, 2020. 